\title{
Impact of High-Altitude Ultraviolet Radiation on Functionability of Flight Crews
}

\author{
Jezdimir Knezevic* \\ Mirce Akademy, Woodbury Park, Exeter, UK
}

*Corresponding author: Jezdimir Knezevic, Mirce Akademy, Woodbury Park, Exeter, UK.

Received Date: July 03, 2019

Published Date: July 09, 2019

\begin{abstract}
The philosophy of MIRCE Science is based on the premise that the purpose for the existence of any functionable system is to do functionability work. The work is done when the expected measurable function is performed through time. In MIRCE Science a flight crew is considered as an element of a flying system type. MIRCE Mechanics is a part of MIRCE Science that focuses on the scientific understanding of the mechanisms of the interactions between functionability elements and the consequences on functionability performance. The research performed shown that pilots and flight crews on aircraft is twice as likely as the general population to develop life-threatening melanoma skin cancer. On average, those who developed melanoma were $42 \%$ more likely to die compared with the general population. With progress in aviation technology, aircraft will fly longer and at higher altitudes attracting more and more passengers demanding more and more flights. Hence, the main objective of this paper is to investigate the mechanism of interactions between the high-altitude ultraviolet radiation on functionability of flight crew.
\end{abstract}

\section{Introduction}

The philosophy of MIRCE Science is based on the premise that the purpose for the existence of any functionable system is to do functionability work. The work is done when the expected measurable function is performed through time [1]. Thus, in MIRCE Science a flight crew is considered to be an element of a functionable system type whose work is done when passengers and cargo are transported through the air between given destinations.

MIRCE Mechanics is a part of MIRCE Science that focuses on the scientific understanding of the mechanisms of the interactions between functionability elements and the consequential impacts on functionability performance. For example, total ultraviolet (UV) radiation is twice as intense at an altitude of 30,000 feet $(10,000$ meters), the cruising height of passenger planes, then it is at ground level. The research performed shows that pilots and flight crews on aircraft are twice as likely as the general population to develop life-threatening melanoma skin cancer. On average, pilots and cabin crew who developed melanoma were $42 \%$ more likely to die compared with the general population [2].

With continuous progress in aviation technology, aircraft will be flying longer and at higher altitudes attracting more and more passengers demanding more and more flights. Hence, the main objective of this paper is to investigate the impact of the highaltitude ultraviolet radiation on functionability of flight crews in commercial aviation.

\section{Ultraviolet Radiation}

Ultraviolet radiation is a type of electromagnetic radiation that comes from the sun and transmitted in waves or particles at different wavelengths and frequencies. This broad range of wavelengths is known as the electromagnetic (EM) spectrum. The spectrum is generally divided into seven regions in order of decreasing wavelength and increasing energy and frequency. The common designations are radio waves, microwaves, infrared (IR), visible light, ultraviolet, $\mathrm{X}$-rays and gamma rays.

The word "ultraviolet" means "beyond violet". The German physicist Johann Wilhelm Ritter discovered ultraviolet radiation in 1801. He noticed invisible light beyond the violet portion of the visible spectrum darkened silver chloride treated paper more quickly than violet light. Ritter called the invisible light "oxidising rays", referring to the chemical activity of the radiation. Most people used the phrase "chemical rays" until the end of the 19th century, when "heat rays" became known as infrared radiation and "chemical rays" became ultraviolet radiation. 
Ultraviolet light falls in the range of the EM spectrum between the visible light and X-rays. It has frequencies of about $8 \times 1014$ to $3 \times 1016$ cycles per second, or hertz ( $\mathrm{Hz})$, and wavelengths of about 380 nanometers to about $10 \mathrm{~nm}$. UV is generally divided into three sub-bands:

- $\quad$ UVA, or near UV (315-380 nm)

- $\quad$ UVB, or middle UV (280-315 nm)

- $\quad$ UVC, or far UV (180-280 nm)

Radiations with wavelengths from $10 \mathrm{~nm}$ to $180 \mathrm{~nm}$ are usually referred to as "vacuum or extreme UV." These wavelengths only propagate in a vacuum, as they are blocked by earth's atmosphere.

Besides the sun, there are numerous celestial sources of UV radiation. Very large young stars shine most of their light in ultraviolet wavelengths. Because Earth's atmosphere blocks much of this UV radiation, particularly at shorter wavelengths, observations are conducted using high-altitude balloons and orbiting telescopes equipped with specialized imaging sensors and filters for observing in the UV region of the EM spectrum. Most observations are conducted using charge-coupled devices (CCD), detectors designed to be sensitive to short-wavelength photons. These observations can determine the surface temperatures of the hottest stars and reveal the presence of intervening gas clouds between the Earth and quasars.

\section{Ionisation}

Ultraviolet radiation has enough energy to break chemical bonds. Due to their higher energies, ultraviolet photons can cause ionization, which is a process in which electrons break away from atoms. The resulting vacancy affects the chemical properties of the atoms and causes them to form or break chemical bonds that they otherwise would not. This can be useful for chemical processing, or it can be damaging to materials and living tissues. This damage can be beneficial, for instance, in disinfecting surfaces. However, ionization can also be harmful, particularly to skin and eyes, which are most adversely affected by higher-energy UVB and UVC radiation.

\section{Ultraviolet effects}

Most of the natural ultraviolet light human's encounter comes from the sun. However, only about $10 \%$ of sunlight is UV, and only about one-third of this penetrates the atmosphere to reach the ground. Of the solar UV energy that reaches the equator, 95\% is UVA and $5 \%$ percent is UVB. No measurable UVC from solar radiation reaches the Earth's surface, because ozone, molecular oxygen and water vapour in the upper atmosphere completely absorb the shortest UV wavelengths.[10] Nevertheless, broad-spectrum ultraviolet radiation (UVA and UVB) is the strongest and most damaging to living things.

\section{Environmental factors that influence the UV level}

The amount of UV radiation reaching the Earth's surface varies widely around the globe and through time. Several factors contribute to this variation at any given location. They are addressed below in order of importance, together with brief explanations [3]:

Cloud cover: It plays a highly influential role in the amount of both UVA and UVB radiation reaching the ground. Each water droplet in a cloud scatters some incoming UV radiation back into space, so a thick cover of clouds protects organisms and materials from almost all UV. The larger the percentage of the sky that is covered by clouds, the less UV reaches the ground. The more opaque the cloud, the less UVB. However, thin or broken cloud cover can be deceiving to people who are sunbathing, and the result can be unexpected and severe sunburn.

Ozone in the stratosphere: It is a gas composed of three oxygen atoms combined in a single molecule (03). It is a naturally generated in the stratosphere where it strongly absorbs incoming UV radiation. However, as stratospheric ozone decreases, UV radiation is allowed to pass through, and exposure at the Earth's surface increases. Exposure to shorter wavelengths increases by a larger percentage than exposure to longer wavelengths.

Oblique angle of sunlight reaching the surface: At any given time, sunlight strikes most of the Earth at an oblique angle. In this way, the number of UV photons is spread over a wider surface area, lowering the amount of incoming radiation at any given spot, compared to its intensity when the sun is directly overhead. In addition, the amount of atmosphere crossed by sunlight is greater at oblique angles than when the sun is directly overhead. Thus, the light travels through more ozone before reaching the Earth's surface, thereby increasing the amount of UVB that is absorbed by molecules of ozone and reducing UVB exposure at the surface. When the sun is directly overhead, forming a $90^{\circ}$ angle with the surface, sunlight is spread over the minimum area. Also, the light only has to pass through the atmosphere directly above the surface. An increased angle between the sun and the surface, due to latitude, time of day, and season, spreads the same amount of energy over a wider area, and the sunlight passes through more atmosphere, diffusing the light. Thus, UVB radiation is stronger at the equator than the poles, stronger at noon than evening and stronger in summer than winter.

Aerosols: Unlike clouds, aerosols in the troposphere, such as dust and smoke, not only scatter but also absorb UVB radiation. Usually the UV reduction by aerosols is only a few percent, but in regions of heavy smoke or dust, aerosol particles can absorb more than $50 \%$ of the radiation. While the presence of aerosols anywhere in the atmosphere will always scatter some UV radiation back to space, in some circumstances, aerosols can contribute to an increase in UV exposure at the surface. For example, over Antarctica, cold temperatures cause ice particles to form in the stratosphere (Polar Stratospheric Clouds). The nuclei for these particles are thought to be sulphuric acid aerosol, possibly of volcanic origin. The ice particles provide the surfaces that allow complex chemical reactions to take place in a manner than can deplete stratospheric ozone [4]. The eruption of an active volcano Mt. Pinatubo, Philippines, in 1991 injected sulphate aerosols into the stratosphere, significantly 
though temporarily depleting stratospheric ozone and resulting in an increase of UVB reaching the Earth's surface. Over millions of years, the biosphere has evolved to deal with temporary increases in UV from reductions in stratospheric ozone by natural causes such as volcanic eruptions.

Elevation: Living organisms at high elevations are generally exposed to more solar radiation and with it, more UVB than organisms at low elevations. This is because at high elevations UVB radiation travels through less atmosphere before it reaches the ground, and so it has fewer chances of encountering radiationabsorbing aerosols or chemical substances (such as ozone and Sulphur dioxide) than it does at lower elevations.

Reflectivity of the earth's surface: As a highly reflective substance, snow dramatically increases UVB exposure near the Earth's surface as it reflects most of the radiation back into the atmosphere, where it is then scattered back toward the surface by aerosols and air molecules. Fresh snow can reflect much as 94 percent of the incoming UV radiation. In contrast, snow-free lands typically reflect only 2-4\% of UV and ocean surfaces reflect about 5-8\%.

\section{Ozone depletion and UV radiation level}

Depletion of the ozone layer is likely to aggravate existing health effects caused by exposure to UV radiation, as stratospheric ozone is a particularly effective UV radiation absorber. As the ozone layer becomes thinner, the protective filter provided by the atmosphere is progressively reduced. Higher UV radiation levels, and especially higher UVB levels will have the greatest impact on humans, animals, marine organisms and plant life.

\section{Impact of UV Radiation on Life on Earth}

UV radiation from the sun has always played important roles in earth's environment and affects most living organisms. Biological actions of many species have evolved to deal with it. However, as UV radiations at different wavelengths have different effects living organisms have to cope with the harmful effects as well as the beneficial ones. The UVA plays a helpful and essential role information of Vitamin D by the skin and plays a harmful role in that it causes sunburn on human skin and cataracts in human eyes. The incoming UVB part of the electromagnetic spectrum causes damage at the molecular level to the fundamental building block of life, deoxyribonucleic acid (DNA).

DNA readily absorbs UVB radiation, which commonly changes the shape of the molecule in one of several ways. Changes in the DNA molecule often mean that protein-building enzymes cannot "read" the DNA code at that point on the molecule, resulting in either creation of distorted proteins or death of cells. However, during the millions of years of evolution, in the presence of UVB radiation, cells have developed the ability to repair DNA. A special enzyme arrives at the damage site, removes the damaged section of DNA, and replaces it with the proper components; govern by the information elsewhere on the DNA molecule. This makes DNA somewhat resilient to damage by UVB.

\section{Some effects of UVB radiation on the biosphere}

Biological scientists would like to be able to demonstrate a direct correlation between the amount of exposure to UVB radiation and the harm it causes. This is an enormously complicated question that depends on many different variables, such as varying degrees of susceptibility among different species, and most of these variables are not yet completely understood. For example, the same organism in different bodies of water in different parts of the ocean may respond differently to UVB increases. Furthermore, stress to organisms and ecosystems from increased exposure to UVB are modified by interactions among many other stresses, such as lack of water or nutrients.

It is known that increased exposure to UVB radiation has specific effects on crops, terrestrial ecosystems, aquatic ecosystems, and biogeochemical cycles. UVB impairs photosynthesis in many species. Overexposure to UVB reduces size, productivity, and quality in many of the crop plant species that have been studied (among them, many varieties of rice, soybeans, winter wheat, cotton, and corn). Similarly, overexposure to UVB impairs the productivity of phytoplankton in aquatic ecosystems. UVB increases plants' susceptibility to disease. Since some species are more vulnerable to UVB than others, an increase in UVB exposure has the potential to cause a shift in species composition and diversity in various ecosystems. Because UVB affects organisms that move nutrients and energy through the biosphere, it is to be expected that changes in their activities will alter biogeochemical cycles. For example, reducing populations of phytoplankton would significantly impact the world's carbon cycle, because phytoplankton stores huge amounts of carbon in the ocean. Overexposure to ultraviolet radiation can change the flowering times of some kinds of plants and therefore will affect the animals that depend on them.

\section{Impact of UV radiation on human skin}

A suntan is a reaction to exposure to harmful UVB rays resulting from the body's natural defence mechanism govern by a pigment called melanin, which is produced by cells in the skin called melanocytes. Melanin absorbs UV light and dissipates it as heat. When the body senses sun damage, it sends melanin into surrounding cells and tries to protect them from sustaining more damage. The pigment causes the skin to darken.

Melanin is a natural sunscreen. However, continued exposure to UV radiation can overwhelm the body's defences. When this happens, a toxic reaction occurs, resulting in sunburn. Ultraviolet rays can damage the DNA in the body's cells. The body senses this destruction and floods the area with blood to help with the healing process. Painful inflammation occurs as well. Sometimes the cells with DNA mutated by the sun's rays turn into problem cells that don't die but keep proliferating as cancers. The UV radiation causes random damages in the DNA and DNA repair process such that cells acquire the ability to avoid dying. People who get sunburned repeatedly are at much higher risk. The risk for the deadliest form of skin cancer, called melanoma, doubles for someone who has received five or more sunburns. 
The effects of UVB radiation on human skin are varied and widespread. UVB induces skin cancer by causing mutation in DNA and suppressing certain activities of the immune system. The United Nations Environment Program estimates that a sustained $1 \%$ depletion of ozone will ultimately lead to a $2-3 \%$ increase in the incidence of non-melanoma skin cancer. UVB may also suppress the body's immune response to Herpes simplex virus and to skin lesion development and may similarly harm the spleen.

\section{Molecular mechanisms of ultraviolet radiation carcinogenesis}

UV radiation is a potent DNA damaging agent and a known inducer of skin cancer in experimental animals. According to Ananthaswamy [5] there is excellent scientific evidence to indicate that most non-melanoma human skin cancers are induced by repeated exposure to sunlight. UV radiation is unique in that it induces DNA damage that differs from the lesions induced by any other carcinogen. The prevalence of skin cancer on sun-exposed body sites in individuals with the inherited disorder XP suggests that defective repair of UV-induced DNA damage can lead to cancer induction. Carcinogenesis in the skin, as elsewhere, is a multistep process in which a series of genetic and epigenetic events leads to the emergence of a clone of cells that have escaped normal growth control mechanisms. The principal candidates that are involved in these events are oncogenes and tumour suppressor genes. Oncogenes display a positive effect on transformation, whereas tumour suppressor genes have an essentially negative effect, blocking transformation. Activated ras oncogenes have been identified in human skin cancers. In most cases, the mutations in the ras oncogenes have been localized to pyrimidine-rich sequences, which indicates that these sites are probably the targets for UVinduced DNA damage and subsequent mutation and transformation. The finding that activation of ras oncogenes in benign and selfregressing keratoacanthomas in both humans and in animals indicates that they play a role in the early stages of carcinogenesis (Corominas et al., 1989) [5]. Since cancers do not arise immediately after exposure to physical or chemical carcinogens, ras oncogenes must remain latent for long periods of time. Tumor growth and progression into the more malignant stages may require additional events involving activation of other oncogenes or deletion of growth suppressor genes. In addition, amplification of protooncogenes or other genes may also be involved in tumor induction or progression. In contrast to the few studies that implicate the involvement of oncogenes in UV carcinogenesis, the role of tumor suppressor genes in UV carcinogenesis is unknown. Since cancerprone individuals, particularly XP patients, lack one or more repair pathways, one can speculate that DNA repair enzymes would confer susceptibility to both spontaneous and environmentally induced cancers. Another potential candidate that can function as a tumour suppressor gene is the normal c-Ha-ras gene. Spandidos and Wilkie (1988) have shown that the normal c-Ha-ras gene can suppress transformation induced by the mutated ras gene

\section{Impact of UV radiation on human vision}

Human body hair and clothing protect them from UVB, but eyes are vulnerable. Common eye problems resulting from over-exposure to UVB include cataracts, snow blindness, and other ailments, both in humans and animals. While many modern sunglasses offer some UV protection, a significant amount of UV can still reach our eyes in a high exposure situation.

\section{Impact of UV Radiation of Flight Crew}

The National Council on Radiation Protection and Measurements reports that among those U.S. workers exposed to radiation while on the job, flight crews get the largest annual effective dose. Flight crews of high-altitude flying aircraft are exposed to elevated levels of UV radiation as a result of their operations in the upper reaches of the troposphere and lower stratosphere. The air density in that portion of the earth's atmosphere is thinner than at sea level and therefore filters less of the dangerous solar radiation. UV radiation increases at about 6-10\% with every 1,000 ft. of altitude. Between 31,000 and 41,000 ft., where most jet aircraft cruise, UV radiation exposure doubles. Additionally, the destruction of stratospheric ozone, by chlorofluorocarbons and other pollutants, may increase UV radiation exposure. This is especially true close to the equator and both poles due to the thinning of the ozone layer in these regions.

According to Meerkotter, the occupational UV radiation exposure for pilots depends on numerous factors, including:

- the entire time a pilot spent in a cockpit,

- the number of flights along specific routes,

- the specific windscreen properties of the aircraft,

- the individual skin type of a pilot,

- $\quad$ sun position during flight from takeoff to landing,

- $\quad$ the day of the year.

However, the most impactful factor is the incidence angle of the sun with the aircraft's windscreen. The intensity of UV radiation inside the cockpit strongly depends on whether direct sun is entering or not. Without direct sun, the diffuse UV radiation inside the cockpit amounts to about $5 \%$ of the ambient UV irradiance outside the aircraft. In cases of low sun when direct radiation can reach the pilot, percentages grow from 50 to $100 \%$.

\section{UV Radiation and Aircraft Windscreen}

The amount of UV radiation absorbed by aircraft windscreens depends on the material used and the general design. In a study conducted by Meerkotter, released in 2007 [4], the transmittance properties of aircraft windscreens were measured for both visible and invisible optical radiation. Transmission measurements were performed on eight aircraft windscreens. Three windscreens were from large commercial jets (MD-88, Airbus A320 and Boeing 727/737); two from commercial, propeller-driven passenger 
planes (Fokker 27 and ATR 42); one from a small private jet (Raytheon Hawker Horizon); and two from small general aviation, single-engine, propeller-driven planes (Beech Bonanza and Cessna 182).

The general aviation aircraft windscreens were plastic (polycarbonate); the others were multilayer (laminated) composite glass. UV transmittance for both glass and plastic windscreens was less than $1 \%$ for UVB radiation. In the UVA portion of the spectrum, transmittance differences increased from $0.41 \%$ to $53.5 \%$, with plastic attenuating more UV radiation than glass. Hence, the plastic windscreens outperform glass by protecting the pilot's eyes from UV radiation.

Dr. A. Chorley of Britain's Civil Aviation Authority and London South Bank University's Department of Primary Care and Public Health conducted research in optometry and ophthalmology. It focused on ocular UV exposure of pilots in airline and offshore helicopter operations on different aircraft types. Inflight data were captured on five return-sector European airline flights and one transatlantic flight from London Gatwick Airport in addition to four helicopter flights from Aberdeen Dyce Airport.

The study found a wide variation in ocular UVA doses during flights. The main factor influencing exposure was the UV transmission of the windscreen, which fell into two distinct profile types. In an aircraft with good UVA blocking properties, ocular exposure was found to be equivalent to that of office exposure and did not exceed international guideline limits regardless of external conditions or flight time. Most aircraft assessed had poor UVA blocking windscreens, which resulted in an ocular exposure to the unprotected eye in excess of international guideline limits. No significant UVB dose was found.

A research team led by Dr. M. Sanlorenzo, measured UV radiation through the acrylic plastic windscreen, $160 \mathrm{~mm}$ thick, in front of the pilot seat of a TBM 850 at ground level, 2,500 ft., 6,000 ft., 10,000 ft., 15,000 ft., 20,000 ft., 25,000 ft. and 30,000 ft. around midday during April at San Jose, California, and Las Vegas. Published in 2015, the study [6] determined that aircraft occupants flying for $57 \mathrm{~min}$. at 30,000 ft. got the same amount of UVA radiation as from a $20 \mathrm{~min}$. tanning bed session.

Sanlorenzo concluded, "Airplane windscreens do not completely block UVA radiation and therefore not enough to protect pilots. UVA transmission inside airplanes can play a role in pilots' increased risk of melanoma. We believe that better UV protection on aircraft windscreens is necessary to offer cabin crew a hazard-free work environment. We strongly recommend the use of sunscreens and periodical skin checks for pilots and cabin crew" [6].

In addition, Sanlorenzo led another team of medical researchers who reviewed more than 3,527 citations from 19 previous studies on melanoma published between 1990 and 2013, reported data from 1943 to 2008 from 11 countries, and included over 266,431 participants. The results of this study, "The Risk of Melanoma in Airline Pilots and Cabin Crew: A Meta-Analysis," published in 2015, indicated that participants in flight-based occupations are twice as likely to contract melanoma than the general public. Furthermore, pilots specifically have a $42 \%$ higher mortality rate from melanoma compared with the general population. Researchers believe that the culprit is likely UV radiation, which can penetrate glass; even the specialty glass used in aircraft windscreens.

\section{Impact of Long-Term UV Exposure on The Eyes of the Flight Crew}

A long-term exposure to solar UV radiation is another significant hazard to pilots due to the damage done to retinal photoreceptors. Research conducted by Chorley between 2008 and 2015 found no ill effects to the eyes caused by UVB and UVC, since these types of UV radiation are filtered out by the upper atmosphere and the windscreens of most aircraft. However, the study showed that UVA, the least energetic form of UV radiation, is the most harmful to a pilot's eyes and eyesight because a higher percentage of it penetrates the cockpit and cabin of an aircraft [7].

Chorley found that, although rare, short, intense exposure to UVA might lead to conditions such as snow blindness and damage to the cornea. He concluded, "There is good evidence that long-term exposure to solar radiation, especially the ultraviolet and blue light components, is a risk factor for cataracts and, to a lesser extent, agerelated degeneration of the retina" [8].

\section{Sunglasses as Eyesight Damage Prevention}

To prevent eyesight damage a pilot can use sunglasses with sufficient UV protection. In an evaluation of 38 sunglasses used by commercial pilots, Chorley found that all conformed to international standards for UV radiation transmittance, offered sufficient UV radiation protection and against the increase in blue light exposure that pilots experience at altitude. However, it is necessary to stress that used sunglasses with scratched lenses were less effective.

The study concluded that lenses manufactured to minimally comply with standards for UV radiation transmittance could result in excess UV radiation to a pilot based on inflight irradiance data. The study recommended an additional requirement of less than $10 \%$ transmittance in the wavelength of $380 \mathrm{~nm}$. An online survey conducted by Chorley of nearly 3,000 pilots in the UK regarding the use of sunglasses and other onboard protective devices to block sunlight revealed that pilots were critical of standard aircraft protection systems such as visors (and other commonly employed devices) to manage bright light. Sunglass use was found to be surprisingly variable. A quarter of those questioned wore sunglasses less than $10 \%$ of the time or not at all. The most common reasons for sunglasses not being used was the sunglass tint interfering with instrument legibility and discomfort from frames, particularly when used in conjunction with required headsets [9].

According to an FAA study, flying over a thick cloud layer or a snowfield with the sun at its zenith increases the exposure to naturally occurring UV. Snow reflects $85 \%$ of visible and UV radiation, while clouds can reflect up to $80 \%$. In such conditions, sunglasses with a closely fitting wraparound frame design are best 
since UV blocking lenses are useless if radiation is allowed to enter the eye from the sides of the frame. A gray, neutral density filter to block $70-85 \%$ of all visible light is recommended to preserve colour discrimination and enhance the ability to quickly adapt to lower light levels.

People often equate sunglasses with UV radiation protection. The FAA's study pointed out that lens tints or colour are not indicative of the UV blocking ability of a lens. While there are no UV radiation standards for clear prescription lens materials, the American Optometric Association recommends that sunglasses block at least $99 \%$ of solar UV radiation below $400 \mathrm{~nm}$. Without UV treatments, clear glass and CR-39 lenses fall short of this mark. Therefore, it is important when purchasing non-prescription, sunglasses to be sure they are accompanied by proper labelling and/or documentation describing their UV protection properties $[10,11]$.

\section{Conclusion}

The philosophy of MIRCE Science is based on the premise that the purpose for the existence of any functionable system is to do functionability work. The work is done when the expected measurable function is performed through time. In MIRCE Science a flight crew is considered as an element of a flying system type. MIRCE Mechanics is a part of MIRCE Science that focuses on the scientific understanding of the mechanisms of the interactions between functionability elements and the consequences on functionability performance.

The information presented in the paper has shown that ultraviolet radiation is an invisible enemy, to human eyes, that can cause long-lasting and potentially deadly consequences for pilots and crewmembers who are repeatedly exposed to higher levels of both UVA and UVB and for longer periods than those working on the ground. These exposure time risks will, for some flight crew, shorten their career span, requiring more pilots to be trained by the operators and potentially lead to future compensation claims for workplace induced illness. Pilots, especially those operating aircraft at high altitudes, need to have suitable protection to mitigate these risks, which have been shown, based in the information obtained from the literature available that pilots and flight crews on aircraft are twice as likely as the general population to develop life-threatening melanoma skin cancer. On average, those who developed melanoma were $42 \%$ more likely to die compared with the general population.
With continuous progress in aviation technology, aircraft will be flying longer and at higher altitudes attracting more and more passengers demanding more and more flights. Hence, the main objective of this paper was to highlight the potential impact of high-altitude ultraviolet radiation exposure and the increasing risk factors on the functionability of flight crews in commercial aviation over the lifetime of the flight crew.

\section{Acknowledgement}

None.

\section{Conflict of Interest}

No conflicts of interest.

\section{References}

1. Knezevic J (2017) The Origin of mirce Science. Mirce Science Exeter UK 232.

2. Sigurdson AJ, Ron E, Cosm1990ic radiation exposure and cancer risk among flight crew, Radiation Epidemiology Branch, Division of Cancer Epidemiology and Genetics, National Cancer Institute, National Institutes of Health, Department of Health and Human Services, Bethesda, Maryland 20892-7238.

3. Herman JR, Celarier E (1997) Earth Surface Reflectivity Climatology at $340 \mathrm{~nm}$ to $380 \mathrm{~nm}$ from TOMS Data. Journal of Geophysical Research 102: 28003-28011.

4. Nakagawara VB, Montgomery RW, Marshall JW (2007) Optical Radiation Transmittance of Aircraft Windscreens and Pilot Vision. Washington, DC: Federal Aviation Administration.

5. Ananthaswamy HN, Pierceall WE (1990) Molecular mechanisms of ultraviolet radiation carcinogenesis. Photochem Photobiol 52(6): 11191136.

6. Sanlorenzo M, Vujic I, Posch C, Cleaver JE, Quaglino P, et al. (2015) The Risk of Melanoma in Pilots and Cabin Crew UV Measurements in Flying Airplanes. JAMA Dermatol 151(4): 450-452.

7. De Gruijl FR, Van Der Leun JC (1994) Estimate of the wavelength dependency of ultraviolet carcinogenesis in humans and its relevance to the risk assessment of a stratospheric ozone depletion. Health Phys 67(4): 319-325.

8. Charley AC, Evans BJ, Benwell MJ (2011) Civilian Pilot Exposure to Ultraviolet and Blue Light and Pilot Use of Sunglasses. Aviat Space Environ Med 82(9): 895-900.

9. Sanlorenzo M, Wehner MR, Linos E, Kornak J, Kainz W, et al. (2015) The risk of melanoma in airline pilots and cabin crew: a meta. JAMA Dermatol 151(1):51-8.

10. Krotkov NA, PK Bhartia, J Herman Z, Ahmad V, Fioletov (2001) Satellite estimation of spectral surface UV irradiance 2: Effect of horizontally homogeneous clouds and snow. Journal of Geophysical Research 106.

11. Vellette P (2018) High-Altitude UV Exposure: Little-Recognized Rissk for Flight Crews. Business \& Commercial Aviation. 